\title{
Scaling of Total Metabolic, Gravitational and Heat Energy of Living Organisms, Earth and Sun
}

\author{
Atanas Todorov Atanasov \\ Department of Physics and Biophysics, Medical Faculty, Trakia University, Stara Zagora, Bulgaria
}

Email address:

atanastod@abv.bg

To cite this article:

Atanas Todorov Atanasov. Scaling of Total Metabolic, Gravitational and Heat Energy of Living Organisms, Earth and Sun. European Journal of Biophysics. Special Issue: Recent Perspectives in Biophysics. Vol. 3, No. 3-1, 2015, pp. 1-10. doi: 10.11648/j.ejb.s.2015030301.11

\begin{abstract}
The gravitational energy, total metabolic energy and heat energy of living organisms, Earth and Sun are scaled. Statistical analyses have shown that nearly a linear relationship between the total metabolic energy per lifespan of Poikilothermic organisms $\left(\mathrm{P}_{\mathrm{ls}}, \mathrm{kJ}\right)$, total heat energy $\left(\mathrm{THE}_{\mathrm{E}}, \mathrm{kJ}\right)$ of the Earth and the body mass $(\mathrm{M}, \mathrm{kg})$ of Poikilotherms and Earth $\left(\mathrm{M}_{\mathrm{E}}, \mathrm{kg}\right)$ in $\log -\log$ plots holds: $\mathrm{P}_{\mathrm{ls}}=1.696 \times 10^{5} \mathrm{M}^{0.949}$ with $\mathrm{R}^{2}=0.996$. A similar relationship between the total metabolic energy of Homoitherms Mammals and Aves $\left(\mathrm{P}_{\mathrm{ls}}, \mathrm{kJ}\right)$, the total heat energy of Sun (emitted over Earth surface per Earth's lifespan) (THE $\mathrm{kJ})$, and body mass $(\mathrm{M}, \mathrm{kg})$ of Mammals, Aves and Earth $\left(\mathrm{M}_{\mathrm{E}}, \mathrm{kg}\right)$ holds: $\mathrm{P}_{\mathrm{ls}}=10.2 \times 10^{5} \mathrm{M}^{1.023}$ with $\mathrm{R}^{2}=0.996$. The metabolic potential of living organisms, gravitational and heat potential of Earth and Sun are scaled too. The gravitational and 'heat' potential of Earth are emerging as a lower limit of lifespan metabolic potentials of unicellular organisms, while the gravitational and 'heat' potential of Sun are emerging as an upper limit of lifespan metabolic potentials of multicellular organisms (Poikilotherms, Mammals and Aves). The relationships between mass-energy characteristics of living organisms, Earth and Sun show that gravitational and heat energy of Earth and Sun determine maximum and minimum total metabolic energy (per lifespan) of living organisms, while the gravitational and 'heat' potentials of Earth and Sun determine their maximum and minimum lifespan metabolic potentials.
\end{abstract}

Keywords: Total Metabolic, Gravitational, Heat, Energy, Earth, Sun

\section{Introduction}

All living organisms in biosphere, from small unicellular prokaryotes to big multicellular animals and plants live in a spatial area of gravitational fields of Earth, Sun, Moon and other planets from Solar System, as well as in the area of their light and heat emission. The gravity is a constant force throughout the evolution of Earth that acts on mass of living organisms and produced weight of the mass. Thus, gravity is fundamental factor which affects the evolution of organisms $[1,2]$. From physical point of view the gravitational fields of Earth and Sun are characterized by their gravitational potentials (Fix, 1995), given by the equations:

$$
\Gamma_{\mathrm{E}}=-\mathrm{GM}_{\mathrm{E}} / \mathrm{R}_{\mathrm{E}}
$$

and

$$
\Gamma_{\mathrm{S}}=-\mathrm{G} \mathrm{M}_{\mathrm{S}} / \mathrm{R}_{\mathrm{ES}}
$$

where $\Gamma_{\mathrm{E}}(\mathrm{J} / \mathrm{kg})$ is the gravitational potential of Earth equal to $0.625 \times 10^{8} \mathrm{~J} / \mathrm{kg}$ on Earth's ground, $\Gamma_{\mathrm{S}}(\mathrm{J} / \mathrm{kg})$ is the gravitational potential of Sun equal to $8.85 \times 10^{8} \mathrm{~J} / \mathrm{kg}$ on Earth's ground, G is the fundamental gravitational constant of Universe $\left(6.673 \times 10^{-11} \mathrm{Nm}^{2} / \mathrm{kg}\right), \mathrm{M}_{\mathrm{E}}$ is the mass of Earth $\left(5.97 \times 10^{24} \mathrm{~kg}\right)$, $\mathrm{M}_{\mathrm{S}}$ is the mass of Sun $\left(1.99 \times 10^{30} \mathrm{~kg}\right), \mathrm{R}_{\mathrm{E}}$ is the radius of Earth $\left(6.4 \times 10^{6} \mathrm{~m}\right)$ or distance $\mathrm{R}_{\mathrm{ES}}$ between Earth and Sun $\left(1.5 \times 10^{11}\right.$ $\mathrm{m})$.

Because the gravitational potential is defined as work of gravitational force to moves unit mass from given point of field to infinity [3], the gravitational energy of given organism in gravitational field of Earth and Sun can be calculated as a product between mass of organisms $(\mathrm{M})$ and gravitational potentials of Earth $\left(\Gamma_{\mathrm{E}}\right)$ and Sun $\left(\Gamma_{\mathrm{S}}\right)$ on Earth's ground:

$$
\mathrm{GE}_{\mathrm{E}}=\Gamma_{\mathrm{E}} \times \mathrm{M}
$$

and

$$
\mathrm{GE}_{\mathrm{S}}=\Gamma_{\mathrm{S}} \times \mathrm{M}
$$

In these equations the gravitational potentials of Earth $\left(\Gamma_{\mathrm{E}}\right)$ and Sun $\left(\Gamma_{\mathrm{S}}\right)$ on Earth's ground have constant values, while the body mass (M) of living organisms vary 23 orders of 
magnitude (from $8.0 \times 10^{-20} \mathrm{~kg}$ in phage to $3.0 \times 10^{3} \mathrm{~kg}$ in big Mammals) and consequently, the corresponding gravitational energy(GE) of organisms varies 23 orders of magnitude too.

The living organisms must have a necessary energy budget to equilibrate the gravitational attractive forces during their development and division. The life metabolism of organisms is expressed by their basal metabolic rate $P_{\text {met }}(\mathrm{J} / \mathrm{s})$ with dimension of power and biological mean of energy exhausted of organism for unit time at standard physiological conditions [4]. The total metabolic energy per lifespan $\mathrm{P}_{\mathrm{ls}}(\mathrm{J})$ of given organism was calculated as a product between basal metabolic rate $\mathrm{P}_{\text {met }}(\mathrm{J} / \mathrm{s})$ and lifespan $\mathrm{T}_{\mathrm{ls}}(\mathrm{s})$ of organism by the equation:

$$
\mathrm{P}_{1 \mathrm{~s}}=\mathrm{P}_{\text {met }} \times \mathrm{T}_{1 \mathrm{~s}}
$$

The systematic approaches of Atanasov [5, 6, 7, 8] has showed that for Poikilotherms, Mammals and Aves, nearly a linear relationship between the total metabolic energy per lifespan $\mathrm{P}_{1 \mathrm{~s}}$ and body mass of organisms $\mathrm{M}$ holds with correlation coefficient $\mathrm{R}^{2}=0.97-0.98$ :

$$
\mathrm{P}_{1 \mathrm{~s}}=\mathrm{P}_{\mathrm{met}} \times \mathrm{T}_{1 \mathrm{~s}}=\mathrm{A}_{\mathrm{ls}} \mathrm{M}
$$

In equation (4) the linear coefficient $\mathrm{A}_{\mathrm{ls}}(\mathrm{J} / \mathrm{kg})$ presents the 'lifespan metabolic potential' of organism (total metabolic energy, consumed per $1 \mathrm{~kg}$ body mass, per lifespan of organism) and can be calculated as:

$$
\mathrm{A}_{\mathrm{ls}}=\mathrm{P}_{1 \mathrm{~s}} / \mathrm{M}
$$

In Unicellular organisms and small water-living Poikilotherms the most metabolic energy may be expended to metabolic functions, development and reproduction, but the terrestrial animals (Poikilotherms, Mammals and Aves) with big mass are required to equip component to support weight and to resist against gravitational force that arise at movement of body. Because of that, the total metabolic energy and metabolic potentials of organisms grow during evolution, which increases their ability to resist gravity. For example, the lifespan metabolic potential grow from about $(2.0-20) \times 10^{5}$ $\mathrm{J} / \mathrm{kg}$ in Unicellular organisms [9] to $(3.0-30) \times 10^{7} \mathrm{~J} / \mathrm{kg}$ in Poikilotherms [5, and to $(3.0-30) \times 10^{8} \mathrm{~J} / \mathrm{kg}$ in Mammals and Aves [1].

The ratio $\left(\mathrm{P}_{1 \mathrm{~s}} / \mathrm{GE}_{\mathrm{E}}\right)$ between total metabolic and gravitational energy of given organism is equal to the ratio between lifespan metabolic potential $\left(\mathrm{A}_{\mathrm{ls}}, \mathrm{J} / \mathrm{kg}\right)$ of organism and gravitational potential $\left(\Gamma_{\mathrm{E}}, \mathrm{J} / \mathrm{kg}\right)$ of the Earth, accordingly equations (2a) and (3):

$$
\mathrm{P}_{1 \mathrm{~s}} / \mathrm{GE}_{\mathrm{E}}=\mathrm{A}_{1 \mathrm{~s}} / \Gamma_{\mathrm{E}}
$$

Considering that $\Gamma_{\mathrm{E}}=0.625 \times 10^{8} \mathrm{~J} / \mathrm{kg}$, and also the cited above values for $A_{l s}$ in other organisms, the ratio $A_{1 s} / \Gamma_{E}$ is smaller than 1.0 in Unicellular organisms, is about 1.0 in Poikilotherms and exceed 1.0 in Mammals and Aves.

In the Sun and main sequence stars with ${ }^{4} \mathrm{He}$ cores, hydrogen nuclei are converted into helium nuclei and the resulting energy is released from the core as heat, which is emitted as photons to the Universe. The Sun heat flux $\left(\mathrm{H}_{\mathrm{S}}\right)$ emitted over the Earth's surface only was estimated to be about $1.8 \times 10^{17} \mathrm{~J} / \mathrm{s}[10,11,12]$. The total surface heat energy $\left(\mathrm{THE}_{\mathrm{S}}\right)$ of the Sun emitted on Earth's surface for time-period equals to the lifespan of Earth $\mathrm{T}_{\mathrm{E}}=1.5 \times 10^{17} \mathrm{~s}$ (about 4.6 milliards years) that can be tentatively estimates as $2.7 \times 10^{34} \mathrm{~J}$ by the equation:

$$
\mathrm{THE}_{\mathrm{S}}=\mathrm{H}_{\mathrm{S}} \times \mathrm{T}_{\mathrm{E}}
$$

The tentative calculation of $\mathrm{THE}_{\mathrm{S}}$ is connected with fact, that the energy output of the Sun has not been constant over time.

The Earth nucleus emitted heat energy to Earth's surface too, because of big difference between surface and nucleus temperature $\left(4000-5000^{\circ} \mathrm{C}\right)$. The main source of internal energy of nucleus is the heating of Earth's nucleus during Earth formation before 4.6 milliard years and the decay of radioactive elements $\left({ }^{235} \mathrm{U},{ }^{238} \mathrm{U},{ }^{232} \mathrm{Th},{ }^{40} \mathrm{~K}\right)$. The secondary sources are the gravitational pressure of Earth's globe and chemical processes in the internal areas of the Earth. The surface heat flux emitted from Earth's nucleus to Earth's surface was estimated to be $\mathrm{H}_{\mathrm{E}}=4.42 \times 10^{13} \mathrm{~J} / \mathrm{s}$ by data of Pollack et al. [13] and Sclater et al. [14], and $3.0 \times 10^{14} \mathrm{~J} / \mathrm{s}$ by data of Davies [15] and Galimov [16]. The emitted total surface heat energy $\left(\mathrm{THE}_{\mathrm{E}}\right)$ for lifespan of Earth $\left(\mathrm{T}_{\mathrm{E}}=\right.$ $1.5 \times 10^{17} \mathrm{~s}$ ) was tentatively estimated to be between $6.63 \times 10^{30} \mathrm{~J}$ and $4.5 \times 10^{31} \mathrm{~J}$ by the equation:

$$
\mathrm{THE}_{\mathrm{E}}=\mathrm{H}_{\mathrm{E}} \times \mathrm{T}_{\mathrm{E}}
$$

Similarly to lifespan metabolic potential $\mathrm{A}_{\mathrm{ls}}(\mathrm{J} / \mathrm{kg})$ we can define the 'heat' potential of heat emission of Sun $\left(A_{S}, J / k g\right)$ and Earth $\left(A_{E}, J / k g\right)$ by the ratio between the total surface heat energy of Sun $\left(\mathrm{THE}_{\mathrm{S}}\right)$ and Earth $\left(\mathrm{THE}_{\mathrm{E}}\right)$ on the Earth's ground and the mass of the Earth $\left(\mathrm{M}_{\mathrm{E}}\right)$ :

$$
\mathrm{A}=\mathrm{THE} / \mathrm{M}_{\mathrm{E}}
$$

The 'heat' potential in eq. (9) expressed the amount of total heat energy per lifespan, per $1 \mathrm{~kg}$ body mass of Earth and is defined as analog of lifespan metabolic potential of living organisms [6, 17]. The Sun and Earth 'heat' potentials can be estimated to be $A_{S}=45.0 \times 10^{8} \mathrm{~J} / \mathrm{kg}$ and respectively $\mathrm{A}_{\mathrm{E}}=$ $0.07 \times 10^{8} \mathrm{~J} / \mathrm{kg}$ using equation (9). In the scientific literature many approaches are directed mainly to study the influence of hypergravity and microgravity on metabolism, morphogenesis and development of living organisms [18, 19]. Only in single works the integrated scale energy of living organisms is compared with the total heat energy of $\mathrm{He}^{4}$ core systems of main-sequence stars [1] and the metabolic potential of animals is compared with the gravitational potential of Earth [6]. In this direction, the aim of this study is $1 /$ to compare the total metabolic energy of living organisms with their gravitational energy and $2 /$ to scale the total metabolic, total heat and gravitational energy of organisms, Earth and Sun on one 'mass-energy' scale.

\section{Data and Methods}

The data for body mass, lifespan and basal metabolic rate of 
16 Unicellular organism, 31 Poikilothermic and 32 Mammalian and Avian species are taken from papers of Fujiwara [1,9] and Atanasov [5] . All data were recalculated in SI metrical system.

The total metabolic energy per lifespan $\mathrm{P}_{1 \mathrm{~s}}(\mathrm{~J})$ of a given organism was calculated as a product between the basal metabolic rate $\mathrm{P}(\mathrm{J} / \mathrm{s})$ and lifespan $\mathrm{T}_{1 \mathrm{~s}}(\mathrm{~s})$ of organism, in correspondence with equation (3).

The lifespan metabolic potential $\mathrm{A}_{\mathrm{ls}}(\mathrm{J} / \mathrm{kg})$ of a given organisms was calculated by equation (5).

The gravitational energy (GE, J) of a given organism was calculated by equations (2a) and (2b), using the data for gravitational potential of Earth $\left(\Gamma_{\mathrm{E}}, \mathrm{J} / \mathrm{kg}\right)$, Sun $\left(\Gamma_{\mathrm{S}}, \mathrm{J} / \mathrm{kg}\right)$ and mass $\mathrm{M}(\mathrm{kg})$ of organism.

The total metabolic energy per lifespan of studied organisms, lifespan metabolic potentials and gravitational energy of organisms in gravitational field of the Earth and the Sun are given on Table 1, Table 2 and Table 3.

Table 1. Data for cell mass M, basal metabolic rate P and lifespan T by Fujiwara [9], and calculated data for total metabolic energy Pls, lifespan metabolic potential Als, and gravitational energy of unicellular organisms (phage*, prokaryotes and eukaryotes) in the gravitational field of Earth GE $E_{E}$ and Sun GE $E_{S}$ $27^{\circ} \mathrm{C}$..

\begin{tabular}{|c|c|c|c|c|c|c|c|}
\hline $\begin{array}{l}\text { Unicellular } \\
\text { organisms }\end{array}$ & $\begin{array}{l}\text { Mass M } \\
\text { kg }\end{array}$ & $\begin{array}{l}\text { Basal } \\
\text { metabolic } \\
\text { rate } P(J / s)\end{array}$ & $\begin{array}{l}\text { Lifespan } \\
T(s)\end{array}$ & $\begin{array}{l}\text { Total metabolic } \\
\text { energy Pls }(\mathrm{kJ})\end{array}$ & $\begin{array}{l}\text { Lifespan metabolic } \\
\text { potential Als } \\
(\mathrm{kJ} / \mathrm{kg})\end{array}$ & $\begin{array}{l}\text { Cell gravitational } \\
\text { energy in Earth } \\
\text { gravitational field } \\
\text { GE }_{\mathrm{E}}(\mathrm{kJ}) \\
\end{array}$ & $\begin{array}{l}\text { Cell gravitational } \\
\text { energy in Sun } \\
\text { gravitational } \\
\text { field } G_{S}(k J) \\
\end{array}$ \\
\hline 1. Stentor & $8 \times 10^{-8}$ & $0.90 \times 10^{-6}$ & $1.65 \times 10^{5}$ & $1.49 \times 10^{-4}$ & $1.86 \times 10^{3}$ & $5.0 \times 10^{-3}$ & $70.8 \times 10^{-3}$ \\
\hline 2. Paramecium & $4 \times 10^{-10}$ & $2.83 \times 10^{-9}$ & $2.73 \times 10^{4}$ & $7.73 \times 10^{-8}$ & $1.93 \times 10^{2}$ & $2.5 \times 10^{-5}$ & $3.54 \times 10^{-4}$ \\
\hline 3. Tetrahymena & $2 \times 10^{-11}$ & $3.1 \times 10^{-10}$ & $1.02 \times 10^{4}$ & $3.16 \times 10^{-9}$ & $1.58 \times 10^{2}$ & $1.25 \times 10^{-6}$ & $1.77 \times 10^{-5}$ \\
\hline 4. Euglena & $8 \times 10^{-12}$ & $0.9 \times 10^{-10}$ & $3.60 \times 10^{4}$ & $3.24 \times 10^{-9}$ & $4.05 \times 10^{2}$ & $5.0 \times 10^{-7}$ & $7.08 \times 10^{-6}$ \\
\hline 5. Chlamydomonas & $4 \times 10^{-12}$ & $0.53 \times 10^{-10}$ & $3.30 \times 10^{4}$ & $1.76 \times 10^{-9}$ & $4.4 \times 10^{2}$ & $2.5 \times 10^{-7}$ & $3.54 \times 10^{-6}$ \\
\hline 6. Bacillus & $4.3 \times 10^{-15}$ & $3.2 \times 10^{-12}$ & $2.01 \times 10^{3}$ & $6.43 \times 10^{-12}$ & $1.50 \times 10^{3}$ & $2.68 \times 10^{-10}$ & $3.80 \times 10^{-9}$ \\
\hline 7. Staphylococcus & $7.8 \times 10^{-16}$ & $0.9 \times 10^{-13}$ & $1.53 \times 10^{3}$ & $1.38 \times 10^{-13}$ & $1.77 \times 10^{2}$ & $4.87 \times 10^{-11}$ & $6.90 \times 10^{-10}$ \\
\hline 8. Shigella & $7.1 \times 10^{-16}$ & $0.83 \times 10^{-13}$ & $1.32 \times 10^{3}$ & $1.10 \times 10^{-13}$ & $1.55 \times 10^{2}$ & $4.44 \times 10^{-11}$ & $6.30 \times 10^{-10}$ \\
\hline 9. Escherichia & $3.9 \times 10^{-16}$ & $0.53 \times 10^{-13}$ & $1.14 \times 10^{3}$ & $6.08 \times 10^{-14}$ & $1.56 \times 10^{2}$ & $2.44 \times 10^{-11}$ & $3.45 \times 10^{-10}$ \\
\hline 10.Diplococcus & $3.8 \times 10^{-16}$ & $0.53 \times 10^{-13}$ & $1.44 \times 10^{3}$ & $7.68 \times 10^{-14}$ & $2.02 \times 10^{2}$ & $2.37 \times 10^{-11}$ & $3.36 \times 10^{-10}$ \\
\hline 11.Hemophilus & $6.1 \times 10^{-17}$ & $1.3 \times 10^{-14}$ & $1.73 \times 10^{3}$ & $2.22 \times 10^{-14}$ & $3.64 \times 10^{2}$ & $3.81 \times 10^{-12}$ & $5.40 \times 10^{-11}$ \\
\hline 12.T2 phage & $4.6 \times 10^{-19}$ & $0.33 \times 10^{-15}$ & $2.58 \times 10^{3}$ & $8.60 \times 10^{-16}$ & $1.87 \times 10^{3}$ & $2.87 \times 10^{-14}$ & $4.07 \times 10^{-14}$ \\
\hline 13.T4 phage & $3.6 \times 10^{-19}$ & $2.80 \times 10^{-16}$ & $1.26 \times 10^{3}$ & $3.53 \times 10^{-16}$ & $0.98 \times 10^{3}$ & $2.25 \times 10^{-14}$ & $3.18 \times 10^{-14}$ \\
\hline 14.Lambda phage & $2.4 \times 10^{-19}$ & $2.10 \times 10^{-16}$ & $2.58 \times 10^{3}$ & $5.33 \times 10^{-16}$ & $2.22 \times 10^{3}$ & $1.50 \times 10^{-14}$ & $2.12 \times 10^{-14}$ \\
\hline 15.T1 phage & $1.4 \times 10^{-19}$ & $1.36 \times 10^{-16}$ & $2.58 \times 10^{3}$ & $3.53 \times 10^{-16}$ & $2.52 \times 10^{3}$ & $8.75 \times 10^{-15}$ & $1.24 \times 10^{-14}$ \\
\hline 16.T7 phage & $8.6 \times 10^{-20}$ & $0.97 \times 10^{-16}$ & $1.41 \times 10^{3}$ & $1.36 \times 10^{-16}$ & $1.58 \times 10^{3}$ & $5.35 \times 10^{-15}$ & $7.61 \times 10^{-15}$ \\
\hline
\end{tabular}

*Mean metabolic rate and time of cells for syntheses one phage.

Table 2. Data for body mass $M$, basal metabolic rate and lifespan T for Poikilotherms at $17^{\circ} \mathrm{C}$ by Atanasov [5], and calculated data for lifespan metabolic potential Als, gravitational energy of organisms in gravitational field of Earth $G E_{E}$ and Sun $G E_{S}$

\begin{tabular}{|c|c|c|c|c|c|c|c|}
\hline Poikilotherms & $\begin{array}{l}\text { Mass M } \\
\text { kg }\end{array}$ & $\begin{array}{l}\text { Basal } \\
\text { metabolic } \\
\text { rate } P(\mathrm{~J} / \mathrm{s})\end{array}$ & $\begin{array}{l}\text { Life } \\
\text { span } \\
\text { T(s) }\end{array}$ & $\begin{array}{l}\text { Total } \\
\text { metabolic } \\
\text { energy Pls } \\
(\mathrm{kJ})\end{array}$ & $\begin{array}{l}\text { Lifespan } \\
\text { metabolic } \\
\text { potential } \\
\text { Als }(\mathrm{J} / \mathrm{kg}) \\
\end{array}$ & $\begin{array}{l}\text { Gravitational energy } \\
\text { in Earth gravitational } \\
\text { field } G_{E}(\mathbf{k J})\end{array}$ & $\begin{array}{l}\text { Gravitational } \\
\text { energy in Sun } \\
\text { gravitational field } \\
\mathrm{GE}_{\mathrm{S}}(\mathrm{kJ}) \\
\end{array}$ \\
\hline 1. Soil warm & $1.0 \times 10^{-6}$ & $0.84 \times 10^{--6}$ & $2.1 \times 10^{8}$ & $1.76 \times 10^{-1}$ & $1.76 \times 10^{5}$ & $6.25 \times 10^{-2}$ & $8.85 \times 10^{-1}$ \\
\hline 2. Ascaris suum & $1.0 \times 10^{-5}$ & $2.8 \times 10^{-5}$ & $1.2 \times 10^{8}$ & $3.36 \times 10^{0}$ & $3.36 \times 10^{5}$ & $6.25 \times 10^{-1}$ & $8.85 \times 10^{0}$ \\
\hline 3. Clymenea mucosa & $1.1 \times 10^{-4}$ & $1.6 \times 10^{-4}$ & $1.8 \times 10^{8}$ & $2.88 \times 10^{1}$ & $2.62 \times 10^{5}$ & $0.69 \times 10^{1}$ & $9.73 \times 10^{1}$ \\
\hline 4. Lepisma saccharina & $1.25 \times 10^{-6}$ & $9.8 \times 10^{-5}$ & $2.07 \times 10^{6}$ & $2.02 \times 10^{-1}$ & $1.62 \times 10^{5}$ & $0.78 \times 10^{-1}$ & $1.1 \times 10^{0}$ \\
\hline 5. Octopus vulgaris & $2.0 \times 10^{-5}$ & $0.31 \times 10^{-4}$ & $2.4 \times 10^{8}$ & $0.74 \times 10^{1}$ & $0.37 \times 10^{5}$ & $1.25 \times 10^{0}$ & $1.77 \times 10^{1}$ \\
\hline 6. Haliotis rufescencs & $3.0 \times 10^{-3}$ & $2.0 \times 10^{-3}$ & $3.0 \times 10^{8}$ & $6.0 \times 10^{2}$ & $2.0 \times 10^{5}$ & $1.875 \times 10^{2}$ & $2.65 \times 10^{3}$ \\
\hline 7. Sea star & $1.0 \times 10^{-2}$ & $2.2 \times 10^{-2}$ & $2.1 \times 10^{8}$ & $0.46 \times 10^{4}$ & $4.6 \times 10^{5}$ & $6.25 \times 10^{2}$ & $8.85 \times 10^{3}$ \\
\hline 8. Spider (Achaeranea) & $0.73 \times 10^{-4}$ & $1.45 \times 10^{-4}$ & $1.5 \times 10^{8}$ & $2.17 \times 10^{1}$ & $2.97 \times 10^{5}$ & $4.56 \times 10^{0}$ & $6.46 \times 10^{1}$ \\
\hline 9. Spider (Phidiphor) & $3.37 \times 10^{-4}$ & $2.8 \times 10^{-4}$ & $1.8 \times 10^{8}$ & $5.04 \times 10^{1}$ & $1.50 \times 10^{5}$ & $2.11 \times 10^{1}$ & $3.0 \times 10^{1}$ \\
\hline 10.Crustacea(Porcellio) & $0.82 \times 10^{-5}$ & $0.44 \times 10^{-4}$ & $3.0 \times 10^{8}$ & $1.32 \times 10^{1}$ & $1.61 \times 10^{5}$ & $0.51 \times 10^{1}$ & $7.26 \times 10^{1}$ \\
\hline 11.Crustasea (Orcomella) & $2.4 \times 10^{-3}$ & $23.5 \times 10^{-4}$ & $3.0 \times 10^{8}$ & $7.02 \times 10^{2}$ & $2.94 \times 10^{5}$ & $1.5 \times 10^{2}$ & $2.1 \times 10^{3}$ \\
\hline 12.Crustacea (Orconectes) & $14 \times 10^{-3}$ & $1.2 \times 10^{-2}$ & $3.0 \times 10^{8}$ & $3.6 \times 10^{3}$ & $2.57 \times 10^{5}$ & $8.75 \times 10^{2}$ & $1.24 \times 10^{4}$ \\
\hline 13.Crustacea(Emerita) & $15 \times 10^{-3}$ & $9.2 \times 10^{-3}$ & $3.0 \times 10^{8}$ & $2.76 \times 10^{3}$ & $1.84 \times 10^{5}$ & $9.37 \times 10^{2}$ & $1.33 \times 10^{4}$ \\
\hline 14.Pisces(Cyprinus) & $7.4 \times 10^{-2}$ & $3.7 \times 10^{-2}$ & $3.6 \times 10^{8}$ & $13.3 \times 10^{3}$ & $1.80 \times 10^{5}$ & $4.62 \times 10^{3}$ & $6.55 \times 10^{4}$ \\
\hline 15.Pisces(Notothenia) & 0.2 & $6.25 \times 10^{-2}$ & $7.2 \times 10^{8}$ & $4.7 \times 10^{4}$ & $2.35 \times 10^{5}$ & $1.25 \times 10^{4}$ & $1.77 \times 10^{5}$ \\
\hline 16.Pisces(Anguilla) & $4.0 \times 10^{-2}$ & $1.97 \times 10^{-2}$ & $3.6 \times 10^{8}$ & $7.1 \times 10^{3}$ & $1.77 \times 10^{5}$ & $2.5 \times 10^{3}$ & $3.54 \times 10^{4}$ \\
\hline 17.Pisces(Ictalurus) & 0.127 & $5.0 \times 10^{-2}$ & $8.4 \times 10^{8}$ & $4.2 \times 10^{4}$ & $3.3 \times 10^{5}$ & $8.0 \times 10^{3}$ & $1.12 \times 10^{5}$ \\
\hline 18.Pisces(Salvelinus) & 0.112 & $1.28 \times 10^{-1}$ & $3.6 \times 10^{8}$ & $4.61 \times 10^{4}$ & $4.11 \times 10^{5}$ & $7.0 \times 10^{3}$ & $1.0 \times 10^{5}$ \\
\hline 19.Pisces(Mugil) & 0.149 & $8.3 \times 10^{-2}$ & $5.4 \times 10^{8}$ & $4.48 \times 10^{4}$ & $3.0 \times 10^{5}$ & $9.3 \times 10^{3}$ & $1.32 \times 10^{5}$ \\
\hline 20.Frog(Rana) & $3.2 \times 10^{-2}$ & $9.86 \times 10^{-3}$ & $1.08 \times 10^{9}$ & $1.06 \times 10^{4}$ & $3.3 \times 10^{5}$ & $2.0 \times 10^{3}$ & $2.83 \times 10^{4}$ \\
\hline 21.Salamandra & $1.34 \times 10^{-2}$ & $5.58 \times 10^{-3}$ & $6.0 \times 10^{8}$ & $3.35 \times 10^{3}$ & $2.5 \times 10^{5}$ & $0.84 \times 10^{3}$ & $1.18 \times 10^{4}$ \\
\hline 22.Reptilia(Anolis) & $5.0 \times 10^{-3}$ & $3.25 \times 10^{-3}$ & $3.0 \times 10^{8}$ & $9.75 \times 10^{2}$ & $1.95 \times 10^{5}$ & $3.125 \times 10^{2}$ & $4.42 \times 10^{3}$ \\
\hline 23.Reptilia(Dipsosaurus) & $6.4 \times 10^{-2}$ & $1.11 \times 10^{-2}$ & $3.0 \times 10^{8}$ & $3.33 \times 10^{3}$ & $0.52 \times 10^{5}$ & $4.0 \times 10^{3}$ & $5.66 \times 10^{4}$ \\
\hline 24.Reptilia(Amphibolurus) & $3.73 \times 10^{-1}$ & $2.9 \times 10^{-1}$ & $3.0 \times 10^{8}$ & $8.70 \times 10^{4}$ & $2.34 \times 10^{5}$ & $2.33 \times 10^{4}$ & $3.3 \times 10^{5}$ \\
\hline
\end{tabular}




\begin{tabular}{|c|c|c|c|c|c|c|c|}
\hline Poikilotherms & $\begin{array}{l}\text { Mass M } \\
\text { kg }\end{array}$ & $\begin{array}{l}\text { Basal } \\
\text { metabolic } \\
\text { rate } P(J / s)\end{array}$ & $\begin{array}{l}\text { Life } \\
\text { span } \\
\text { T(s) }\end{array}$ & $\begin{array}{l}\text { Total } \\
\text { metabolic } \\
\text { energy Pls } \\
\text { (kJ) } \\
\end{array}$ & $\begin{array}{l}\text { Lifespan } \\
\text { metabolic } \\
\text { potential } \\
\text { Als }(\mathrm{J} / \mathrm{kg}) \\
\end{array}$ & $\begin{array}{l}\text { Gravitational energy } \\
\text { in Earth gravitational } \\
\text { field } \mathbf{G E}_{\mathrm{E}}(\mathbf{k J})\end{array}$ & $\begin{array}{l}\text { Gravitational } \\
\text { energy in Sun } \\
\text { gravitational field } \\
\text { GE }_{\mathrm{S}}(\mathrm{kJ}) \\
\end{array}$ \\
\hline 25.Tortoise(Pseudemys) & $2.5 \times 10^{-1}$ & $1.56 \times 10^{-1}$ & $9.0 \times 10^{8}$ & $1.4 \times 10^{5}$ & $5.60 \times 10^{5}$ & $1.56 \times 10^{4}$ & $2.2 \times 10^{5}$ \\
\hline 26.Sauria(Iguana) & $7.85 \times 10^{-1}$ & $6.8 \times 10^{-1}$ & $6.0 \times 10^{8}$ & $4.08 \times 10^{5}$ & $5.2 \times 10^{5}$ & $4.9 \times 10^{4}$ & $6.95 \times 10^{5}$ \\
\hline 27.Crocodile(Alligator) & 49.0 & $2.12 \times 10^{1}$ & $1.2 \times 10^{9}$ & $2.54 \times 10^{7}$ & $5.18 \times 10^{5}$ & $3.06 \times 10^{6}$ & $4.34 \times 10^{7}$ \\
\hline 28.Natrix & $8.4 \times 10^{-2}$ & $0.33 \times 10^{-1}$ & $4.2 \times 10^{8}$ & $1.39 \times 10^{4}$ & $1.65 \times 10^{5}$ & $5.25 \times 10^{3}$ & $7.43 \times 10^{4}$ \\
\hline 29.Boidae & 1.0 & $1.16 \times 10^{-1}$ & $9.0 \times 10^{8}$ & $1.04 \times 10^{5}$ & $1.04 \times 10^{5}$ & $6.25 \times 10^{4}$ & $8.85 \times 10^{5}$ \\
\hline 30.Piton & 5.0 & $1.97 \times 10^{-1}$ & $9.0 \times 10^{8}$ & $1.77 \times 10^{5}$ & $0.35 \times 10^{5}$ & $3.125 \times 10^{4}$ & $4.42 \times 10^{5}$ \\
\hline 31.Boa & 10.0 & $11.6 \times 10^{-1}$ & $3.0 \times 10^{8}$ & $3.48 \times 10^{5}$ & $0.35 \times 10^{5}$ & $6.25 \times 10^{5}$ & $8.85 \times 10^{6}$ \\
\hline
\end{tabular}

Table 3. Data for body mass $M$, basal metabolic rate $P$ and lifespan T for Homoitherms (Mammals and Aves) at $37^{\circ} \mathrm{C}$ by Fujiwara [1], and calculated data for total metabolic energy Pls, lifespan metabolic potential Als and gravitational energy of organisms in gravitational field of Earth GE $E_{E}$ and Sun GE

\begin{tabular}{|c|c|c|c|c|c|c|c|}
\hline $\begin{array}{l}\text { Homoitherms } \\
\text { (mammals and } \\
\text { aves) }\end{array}$ & $\begin{array}{l}\text { Mass M } \\
\text { kg }\end{array}$ & $\begin{array}{l}\text { Basal } \\
\text { metabolic } \\
\text { rate } P(J / s)\end{array}$ & $\begin{array}{l}\text { Lifespan } \\
T(s)\end{array}$ & $\begin{array}{l}\text { Total metabolic } \\
\text { energy Pls }(k J)\end{array}$ & $\begin{array}{l}\text { Lifespan } \\
\text { metabolic } \\
\text { potential Als } \\
(\mathrm{J} / \mathrm{kg}) \\
\end{array}$ & $\begin{array}{l}\text { Gravitational energy } \\
\text { in Earth } \\
\text { gravitational field } \\
\mathbf{G E}_{\mathbf{E}}(\mathbf{k J})\end{array}$ & $\begin{array}{l}\text { Gravitational energy } \\
\text { in Sun gravitational } \\
\text { field } \mathbf{G E}_{\mathrm{S}}(\mathrm{kJ})\end{array}$ \\
\hline 1. Mouse & $2.2 \times 10^{-2}$ & $1.53 \times 10^{--1}$ & $4.5 \times 10^{7}$ & $0.069 \times 10^{5}$ & $3.136 \times 10^{5}$ & $1.375 \times 10^{3}$ & $0.195 \times 10^{5}$ \\
\hline 2. American mole & $4.0 \times 10^{-2}$ & $0.4 \times 10^{0}$ & $1.05 \times 10^{8}$ & $0.42 \times 10^{5}$ & $10.5 \times 10^{5}$ & $2.5 \times 10^{3}$ & $0.354 \times 10^{5}$ \\
\hline 3. Little brown rat & $1.0 \times 10^{-1}$ & $1.1 \times 10^{-1}$ & $3.0 \times 10^{8}$ & $0.33 \times 10^{5}$ & $3.3 \times 10^{5}$ & $6.25 \times 10^{3}$ & $0.885 \times 10^{5}$ \\
\hline 4. Grownd squirrel & $1.0 \times 10^{-1}$ & $0.47 \times 10^{0}$ & $2.7 \times 10^{8}$ & $1.26 \times 10^{5}$ & $12.6 \times 10^{5}$ & $6.25 \times 10^{3}$ & $0.885 \times 10^{5}$ \\
\hline 5. Golden hamster & $1.2 \times 10^{-1}$ & $0.6 \times 10^{0}$ & $1.17 \times 10^{7}$ & $0.07 \times 10^{5}$ & $5.85 \times 10^{5}$ & $7.5 \times 10^{3}$ & $1.06 \times 10^{5}$ \\
\hline 6. Rat & $2.8 \times 10^{-1}$ & $1.67 \times 10^{0}$ & $9.0 \times 10^{7}$ & $1.50 \times 10^{5}$ & $5.36 \times 10^{5}$ & $1.75 \times 10^{4}$ & $2.48 \times 10^{5}$ \\
\hline 7. Pigeon & $3.8 \times 10^{-1}$ & $1.2 \times 10^{0}$ & $6.0 \times 10^{8}$ & $7.2 \times 10^{5}$ & $18.9 \times 10^{5}$ & $2.375 \times 10^{4}$ & $3.36 \times 10^{5}$ \\
\hline 8. Owl monkey & $4.8 \times 10^{-1}$ & $1.33 \times 10^{0}$ & $3.6 \times 10^{8}$ & $4.8 \times 10^{5}$ & $10 \times 10^{5}$ & $3.0 \times 10^{4}$ & $4.25 \times 10^{5}$ \\
\hline 9. Squirrel monkey & $6.1 \times 10^{-1}$ & $2.77 \times 10^{0}$ & $6.0 \times 10^{8}$ & $1.66 \times 10^{6}$ & $27 \times 10^{5}$ & $3.81 \times 10^{4}$ & $5.4 \times 10^{5}$ \\
\hline 10.Hedgehog & $7.9 \times 10^{-1}$ & $3.2 \times 10^{1}$ & $1.8 \times 10^{8}$ & $5.76 \times 10^{5}$ & $7.3 \times 10^{5}$ & $4.93 \times 10^{4}$ & $7.0 \times 10^{5}$ \\
\hline 11.Guinea pig & $1.0 \times 10^{0}$ & $0.43 \times 10^{1}$ & $2.28 \times 10^{8}$ & $9.88 \times 10^{5}$ & $9.88 \times 10^{5}$ & $6.25 \times 10^{4}$ & $8.85 \times 10^{5}$ \\
\hline 12.Opossum & $1.7 \times 10^{0}$ & $0.47 \times 10^{1}$ & $1.5 \times 10^{8}$ & $7.0 \times 10^{5}$ & $4.11 \times 10^{5}$ & $1.06 \times 10^{5}$ & $15 \times 10^{5}$ \\
\hline 13.Hen & $2.0 \times 10^{0}$ & $0.73 \times 10^{1}$ & $3.0 \times 10^{8}$ & $2.2 \times 10^{6}$ & $11 \times 10^{5}$ & $1.25 \times 10^{5}$ & $17.7 \times 10^{5}$ \\
\hline 14. Rabbit & $3.0 \times 10^{0}$ & $0.57 \times 10^{1}$ & $6.0 \times 10^{8}$ & $3.4 \times 10^{6}$ & $11.3 \times 10^{5}$ & $1.875 \times 10^{5}$ & $26.5 \times 10^{5}$ \\
\hline 15.Echidna & $3.0 \times 10^{0}$ & $0.37 \times 10^{1}$ & $6.0 \times 10^{8}$ & $2.2 \times 10^{6}$ & $7.3 \times 10^{5}$ & $1.875 \times 10^{5}$ & $26.5 \times 10^{5}$ \\
\hline 16.Cat & $3.3 \times 10^{0}$ & $0.8 \times 10^{1}$ & $8.4 \times 10^{8}$ & $6.72 \times 10^{6}$ & $20.3 \times 10^{5}$ & $2.06 \times 10^{5}$ & $29 \times 10^{5}$ \\
\hline 17.Arctic fox & $3.4 \times 10^{0}$ & $0.97 \times 10^{1}$ & $4.2 \times 10^{8}$ & $4.06 \times 10^{6}$ & $11.9 \times 10^{5}$ & $2.125 \times 10^{5}$ & $30 \times 10^{5}$ \\
\hline 18.Red fox & $4.2 \times 10^{0}$ & $1.23 \times 10^{1}$ & $2.94 \times 10^{8}$ & $3.63 \times 10^{6}$ & $8.6 \times 10^{5}$ & $2.625 \times 10^{5}$ & $37 \times 10^{5}$ \\
\hline 19. Raccon & $4.3 \times 10^{0}$ & $0.9 \times 10^{1}$ & $4.2 \times 10^{8}$ & $3.78 \times 10^{6}$ & $8.8 \times 10^{5}$ & $2.687 \times 10^{5}$ & $38 \times 10^{5}$ \\
\hline 20.Goose & $5.0 \times 10^{0}$ & $1.53 \times 10^{1}$ & $12 \times 10^{8}$ & $18.4 \times 10^{6}$ & $36.8 \times 10^{5}$ & $3.125 \times 10^{5}$ & $44 \times 10^{5}$ \\
\hline 21.Rhesus monkey & $6.8 \times 10^{0}$ & $1.6 \times 10^{1}$ & $8.7 \times 10^{8}$ & $13.9 \times 10^{6}$ & $20.44 \times 10^{5}$ & $4.25 \times 10^{5}$ & $60 \times 10^{5}$ \\
\hline 22.Condor & $1.5 \times 10^{1}$ & $1.8 \times 10^{1}$ & $15 \times 10^{8}$ & $27 \times 10^{6}$ & $18 \times 10^{5}$ & $9.37 \times 10^{5}$ & $133 \times 10^{5}$ \\
\hline 23.Dog & $1.3 \times 10^{1}$ & $2.93 \times 10^{1}$ & $4.5 \times 10^{8}$ & $13.2 \times 10^{6}$ & $10.15 \times 10^{5}$ & $8.12 \times 10^{5}$ & $115 \times 10^{5}$ \\
\hline 24.Casuarius & $2.0 \times 10^{1}$ & $1.93 \times 10^{1}$ & $7.5 \times 10^{8}$ & $14.5 \times 10^{6}$ & $7.25 \times 10^{5}$ & $1.25 \times 10^{6}$ & $177 \times 10^{5}$ \\
\hline 25.Goat & $2.8 \times 10^{1}$ & $2.87 \times 10^{1}$ & $6.0 \times 10^{8}$ & $17.2 \times 10^{6}$ & $6.14 \times 10^{5}$ & $1.75 \times 10^{6}$ & $248 \times 10^{5}$ \\
\hline 26.Gray wolf & $3.6 \times 10^{1}$ & $6.33 \times 10^{1}$ & $4.8 \times 10^{8}$ & $30.4 \times 10^{6}$ & $8.44 \times 10^{5}$ & $2.26 \times 10^{6}$ & $320 \times 10^{5}$ \\
\hline 27.Chimpanzee & $5.2 \times 10^{1}$ & $7.0 \times 10^{1}$ & $15 \times 10^{8}$ & $10.5 \times 10^{7}$ & $20.2 \times 10^{5}$ & $3.25 \times 10^{6}$ & $460 \times 10^{5}$ \\
\hline 28.Sheep & $5.6 \times 10^{1}$ & $7.3 \times 10^{1}$ & $6.0 \times 10^{9}$ & $4.4 \times 10^{7}$ & $7.85 \times 10^{5}$ & $3.5 \times 10^{6}$ & $495 \times 10^{5}$ \\
\hline 29.Man & $6.5 \times 10^{1}$ & $6.7 \times 10^{1}$ & $1.8 \times 10^{9}$ & $12 \times 10^{7}$ & $18.46 \times 10^{5}$ & $4.0 \times 10^{6}$ & $575 \times 10^{5}$ \\
\hline 30.Horse & $4.5 \times 10^{2}$ & $4.33 \times 10^{2}$ & $1.1 \times 10^{9}$ & $4.81 \times 10^{8}$ & $10.69 \times 10^{5}$ & $2.81 \times 10^{7}$ & $39.8 \times 10^{7}$ \\
\hline 31.Cow & $4.7 \times 10^{2}$ & $3.3 \times 10^{2}$ & $9.0 \times 10^{8}$ & $29.7 \times 10^{7}$ & $6.32 \times 10^{5}$ & $2.94 \times 10^{7}$ & $41.6 \times 10^{7}$ \\
\hline 32.Elephant & $2.5 \times 10^{3}$ & $1.53 \times 10^{3}$ & $2.04 \times 10^{9}$ & $31.3 \times 10^{8}$ & $12.5 \times 10^{5}$ & $1.56 \times 10^{8}$ & $22.1 \times 10^{8}$ \\
\hline
\end{tabular}

The gravitational energy of Earth $\left(\mathrm{GE}_{\text {Earth }}, \mathrm{J}\right)$ was calculated using the data for gravitational potential $\left(\Gamma_{\mathrm{E}}, \mathrm{J} / \mathrm{kg}\right)$ and mass $\left(\mathrm{M}_{\mathrm{E}}, \mathrm{kg}\right)$ of the Earth seen as spheroid [20]:

$$
\mathrm{GE}_{\text {Earth }}=3 / 5\left(\Gamma_{\mathrm{E}} \times \mathrm{M}_{\mathrm{E}}\right)=2.238 \times 10^{32} \mathrm{~J}
$$

The total heat energy income from the Sun $\left(\mathrm{THE}_{\mathrm{S}}, \mathrm{J}\right)$ was estimated by equation (7) using data for the Sun heat flux on Earth's surface $\left(\mathrm{H}_{S}, J\right)$ and lifespan $\left(\mathrm{T}_{E}, \mathrm{~s}\right)$ of the Earth by Colozza [11] and Gueymard [12].

The total heat energy of the Earth $\left(\mathrm{THE}_{\mathrm{E}}, \mathrm{J}\right)$ was estimated by equation (8) using data for surface heat flux $\left(\mathrm{H}_{\mathrm{E}}, \mathrm{J} / \mathrm{s}\right)$ and lifespan $\left(T_{E}, s\right)$ of the Earth by Davies [15], Galimov [16], and 
Pollack et al.. [13].

The 'heat' potential of heat emission of Sun $\left(A_{S}, J / k g\right)$ and Earth $\left(A_{E}, J / k g\right)$ was calculated by the ratio (9).
All data for the Earth, Sun and planets of the Solar System are summarized in Table 4.

Table 4. Mass M, lifespan Tls and gravitational potentials $\Gamma$ of planets estimated for minimum distance to Earth accordingly data by Fix [41]. The heat fluxes $H$ and estimated total heat energy THE of Earth and Sun are given too.

\begin{tabular}{llllll}
\hline $\begin{array}{l}\text { COSMIC } \\
\text { OBJECTS }\end{array}$ & $\begin{array}{l}\text { Mass of objects } \\
\mathbf{M ~ k g}\end{array}$ & Lifespan Tls $(\mathbf{s})$ & $\begin{array}{l}\text { Gravitational potentials on } \\
\text { Earth surface } \boldsymbol{\Gamma}(\mathbf{k J} / \mathbf{k g})\end{array}$ & $\begin{array}{l}\text { Heat flux on Earth } \\
\text { surface } \mathbf{H}(\mathbf{J} / \mathbf{s})\end{array}$ & $\begin{array}{l}\text { Total heat energy emitted on } \\
\text { Earth surface THE }(\mathbf{J})\end{array}$ \\
\hline 1.Earth & $5.97 \times 10^{24}$ & $1.5 \times 10^{17}$ & $0.625 \times 10^{5}$ & $3.0 \times 10^{14}$ & $4.5 \times 10^{31}$ \\
2.Sun & $1.99 \times 10^{30}$ & $1.5 \times 10^{17}$ & $8.85 \times 10^{5}$ & $1.8 \times 10^{17}$ & $2.7 \times 10^{34}$ \\
3.Mercury & $3.28 \times 10^{23}$ & $1.5 \times 10^{17}$ & $2.37 \times 10^{-1}$ & & \\
4. Venus & $4.86 \times 10^{24}$ & $1.5 \times 10^{17}$ & 7.72 & \\
5. Mars & $0.638 \times 10^{24}$ & $1.5 \times 10^{17}$ & $5.45 \times 10^{-1}$ & \\
6. Jupiter & $1.897 \times 10^{27}$ & $1.5 \times 10^{17}$ & $2.1 \times 10^{2}$ & \\
7. Saturn & $5.68 \times 10^{26}$ & $1.5 \times 10^{17}$ & $2.0 \times 10^{1}$ & \\
8. Uran & $8.69 \times 10^{25}$ & $1.5 \times 10^{17}$ & 2.14 & & \\
9. Neptun & $1.029 \times 10^{26}$ & $1.5 \times 10^{17}$ & 1.57 & & \\
10. Pluto & $0.656 \times 10^{24}$ & $1.5 \times 10^{17}$ & $7.6 \times 10^{-3}$ & \\
11. Moon & $7.35 \times 10^{22}$ & $3.84 \times 10^{8}$ & $1.276 \times 10^{1}$ & & \\
\hline
\end{tabular}

Statistical software 'STATISTICA' was used for calculation of allometric relationships between the total metabolic energy, total heat energy and gravitational energy of the living organisms, Earth and Sun. A least-squares regression analyses was used in all calculations.

\section{Result and Discussion}

The body mass of all studied organisms (Unicellular, Pokilotherms, Mammals and Aves) vary 23 orders of magnitude, from $8.0 \times 10^{-20} \mathrm{~kg}$ in unicellular organisms to about $3.0 \times 10^{3} \mathrm{~kg}$ in Mammals. The total metabolic and gravitational energy of studied organisms vary 25 orders of magnitude, from about $1.0 \times 10^{-16} \mathrm{~J}$ in unicellular organisms to about $1.0 \times 10^{9} \mathrm{~J}$ in Mammals and Aves. The lifespan vary 5 orders of magnitude, from about $1.0 \times 10^{3} \mathrm{~s}$ in unicellular organisms to about $1.0 \times 10^{8} \mathrm{~s}$ in Mammals and Aves. The large magnitude of variation between body mass, lifespan and energetic characteristics of organisms allows study the problems statistically. The comparing of lifespan metabolic potential
$\left(\mathrm{A}_{\mathrm{ls}}, \mathrm{kJ} / \mathrm{kg}\right)$ of living organisms with gravitational potential $(\Gamma$, $\mathrm{kJ} / \mathrm{kg}$ ) of Sun and planets from Solar Systems shows that the metabolic potentials of organisms and gravitational potentials of Sun, Earth and giant planet Jupiter fall in the same range of magnitude and values of characteristics (Figure 1). The lifespan metabolic potentials of all studied organisms on Fig.1 varied 4-5 orders of magnitude, from $1.0 \times 10^{2} \mathrm{~kJ} / \mathrm{kg}$ in bacteria to $1.0 \times 10^{6} \mathrm{~kJ} / \mathrm{kg}$ in Mammals and Aves, and are located between gravitational potential of Jupiter $\left(\Gamma_{\mathrm{J}}=2.1 \times 10^{2} \mathrm{~kJ} / \mathrm{kg}\right)$ and gravitational potential of Sun $\left(\Gamma_{\mathrm{S}}=8.85 \times 10^{5} \mathrm{~kJ} / \mathrm{kg}\right)$. The gravitational potential of Earth $\left(\Gamma_{\mathrm{E}}=0.625 \times 10^{5} \mathrm{~kJ} / \mathrm{kg}\right)$ occupies an intermediate position. The nearly $1.0 \times 10^{5}$ fold smaller gravitational potentials $\left(1.0 \times 10^{-3} \mathrm{~kJ} / \mathrm{kg}-1.0 \times 10^{1} \mathrm{~kJ} / \mathrm{kg}\right)$ of other planets from the Solar System given on Table 4 (Mercury, Venus, Mars, Saturn, Uran, Neptun and Pluto), in comparison to lifespan metabolic potentials $\left(1.0 \times 10^{2} \mathrm{~kJ} / \mathrm{kg}-\right.$ $1.0 \times 10^{6} \mathrm{~kJ} / \mathrm{kg}$ ) of living organisms show that these planets are unable to provide direct gravitational influence over living organisms on Earth's surface.



Figure 1. Lifespan metabolic potential $A_{l s}(\mathrm{~kJ} / \mathrm{kg})$ of unicellular and multicellular organisms, comparing with gravitational potentials (I, $\left.\mathrm{kJ} / \mathrm{kg}\right)$ of Sun, Earth, Jupiter and Saturn on Earth's ground. 
The lifespan metabolic potential $\left(\mathrm{A}_{\mathrm{ls}}\right)$ of Unicellular organisms on Fig. 1 with mass $8.0 \times 10^{-20} \mathrm{~kg}-8.0 \times 10^{-8} \mathrm{~kg}$ range in diapason of $1.0 \times 10^{2} \mathrm{~kJ} / \mathrm{kg}-2.5 \times 10^{3} \mathrm{~kJ} / \mathrm{kg}$. The minimum metabolic potential $\left(1.0 \times 10^{2} \mathrm{~kJ} / \mathrm{kg}\right)$ of these organisms is comparable to $1 / 2$ of Jupiter gravitational potential $\left(1 / 2 \Gamma_{\mathrm{J}}\right.$ $=1.05 \times 10^{2} \mathrm{~kJ} / \mathrm{kg}$ ), while their maximum metabolic potential $\left(2.5 \times 10^{3} \mathrm{~kJ} / \mathrm{kg}\right)$ exceeds 10 fold gravitational potential of Jupiter $\left(\Gamma_{\mathrm{J}}=2.1 \times 10^{2} \mathrm{~kJ} / \mathrm{kg}\right)$, but does not reach the gravitational potential of Earth $\left(\Gamma_{\mathrm{E}}=0.625 \times 10^{5} \mathrm{~kJ} / \mathrm{kg}\right)$. Thus, the full diapason of metabolic potentials of Unicellular organisms vary between $1 / 2 \Gamma_{\mathrm{J}}$ and $1 / 2 \Gamma_{\mathrm{E}}$.

The ratio $\left(\mathrm{A}_{\mathrm{ls}} / \Gamma_{\mathrm{E}}\right)$ between lifespan metabolic potentials of Unicellular organisms and gravitational potential of Earth range between $1.0 \times 10^{-2}-1.0 \times 10^{-3}$ fold and is equal to the ratio between the total metabolic and gravitational energy of the organisms $\left(\mathrm{A}_{\mathrm{ls}} \mathrm{M} / \Gamma_{\mathrm{E}} \mathrm{M}\right)$. The $10^{2}-10^{3}$ fold smaller total metabolic energy $\left(\mathrm{A}_{\mathrm{ls}} \mathrm{M}\right)$ of Unicellular in comparison to their gravitational energy $\left(\Gamma_{\mathrm{E}} \mathrm{M}\right)$ shows that the life processes in these organisms doesn't depend on Earth gravitation. Although their total metabolic energy is hundred of thousands times smaller than their gravitational energy, they are appeared and lived for a billions years ago under the influence of the Earth gravitation without significant changes in their cellular characteristics. Actually, in the scientific literature predominates the concept, that the Unicellular organisms are not presumed to be gravity sensing $[21,22,23]$. The experiments on growth of Unicellular on hypergravity and microgravity conditions confirm this concept. The study of Deguchi et al. [23] showed that microbial growth is possible up to hyperaccelerations $\sim 10^{5} \times \mathrm{g}\left(\mathrm{g}=9.8 \mathrm{~m} / \mathrm{s}^{2}\right)$. The small hyperacceleration to about $\sim 10 \times \mathrm{g}$ can not affect growth of Gram-negative prokaryotes (E. coli, Paracoccus denitrificans, Shewanella amazonensis) and Gram-positive prokaryotes (Lactobacillus delbrueckii). Similar observations were reported for eukaryotic Paramecium tetraurelia and Saccharomyces cerevisiae. On hypergravity up to $\sim 20 \times \mathrm{g}$ these Eukaryotes only significantly decrease proliferation rate and population density accordingly data by Kato, Mogami and Baba [24]. On the contrary, growth of Unicellular Prokaryotes and Eukaryotes on microgravity enhance their growth, gene expression, virulence and secondary metabolism accordingly data by Demain and Fang [25], Nickerson et al. [26], Purevdorj-Gage et al.[27], and Horneck, Klaus and Mancinelli [28].

From present on Table 4 planets solely the giant planet Jupiter creates significant gravitational potential on the Earth's ground. Any authors, like Ksanfomaliti [29] from aesthetic considerations underlining the important role of giant planets, like Jupiter, for the genesis of the life processes on the Earth. This author noted cautiously, that there even existed variants of the anthropic principle stating that the appearance and development of life on the Earth owes itself to Jupiter. However, Jupiter's satellite Europe has been identified as a potential site for the extra-terrestrial search for life in the Solar System [30, 31].

The next evolutionary group on Fig.1 is represented by milticellular Poikilothermic organisms with mass $1.0 \times 10^{-6} \mathrm{~kg}$ $50 \mathrm{~kg}$ and lifespan metabolic potentials in diapason of $3.0 \times 10^{4}$ $\mathrm{kJ} / \mathrm{kg}-6.0 \times 10^{5} \mathrm{~kJ} / \mathrm{kg}$. The minimum metabolic potential of Poikilotherms $\left(\mathrm{A}_{\mathrm{ls}}=3.0 \times 10^{4} \mathrm{~kJ} / \mathrm{kg}\right)$ is near to $1 / 2$ gravitational potential of the Earth $\left(1 / 2 \Gamma_{\mathrm{E}}=3.175 \times 10^{4} \mathrm{~kJ} / \mathrm{kg}\right)$, while their maximum metabolic potentials $\left(\mathrm{A}_{\mathrm{ls}}=6.0 \times 10^{5} \mathrm{~kJ} / \mathrm{kg}\right)$ exceed about 10 fold gravitational potential of Earth and is approaching to the gravitational potential of Sun $\left(\Gamma_{\mathrm{S}}=\right.$ $8.85 \times 10^{5} \mathrm{~kJ} / \mathrm{kg}$ ). The full diapason of lifespan metabolic potentials of Poikilotherms varies predominantly between $1 / 2$ $\Gamma_{\mathrm{E}}$ and $1 / 2 \Gamma_{\mathrm{S}}$ and these values can be regarded as upper and lower limit of the metabolic potentials of Poikilotherms.

The ratios $\left(\mathrm{A}_{\mathrm{ls}} / \Gamma_{\mathrm{E}}\right)$ between lifespan metabolic potentials of Poikilothermic organisms and gravitational potential of the Earth range between 0.5 and 10 . This shows that the total metabolic energy $\left(\mathrm{A}_{\mathrm{Is}} \mathrm{M}\right)$ of Poikilotherms exceeds considerably their gravitational energy $\left(\Gamma_{\mathrm{E}} \mathrm{M}\right)$, which allows them to overcome the Earth gravity in the water and on the ground despite their big body mass.

The next evolutionary group on Fig. 1 is represented by the complexly arranged Mammals and Aves with mass $2.0 \times 10^{-2}$ $\mathrm{kg}-2.5 \times 10^{3} \mathrm{~kg}$ and lifespan metabolic potentials in diapason of $3.0 \times 10^{5} \mathrm{~kJ} / \mathrm{kg}-3.5 \times 10^{6} \mathrm{~kJ} / \mathrm{kg}$. The minimum metabolic potential of Mammals and Aves $\left(3.0 \times 10^{5} \mathrm{~kJ} / \mathrm{kg}\right)$ is near to $1 / 2$ Sun gravitational potential $\left(1 / 2 \Gamma_{\mathrm{S}}=4.42 \times 10^{5} \mathrm{~kJ} / \mathrm{kg}\right)$, while the maximum metabolic potential $\left(3.5 \times 10^{6} \mathrm{~kJ} / \mathrm{kg}\right)$ exceed 4 fold $\Gamma_{\mathrm{S}}$. Thus, the $1 / 2 \Gamma_{\mathrm{S}}$ and $\Gamma_{\mathrm{S}}$ are located near to lower and upper limits of the lifespan metabolic potentials of Mammals and Aves. The $\left(\mathrm{A}_{\mathrm{IS}} / \Gamma_{\mathrm{E}}\right)$ ratio, and respectively $\left(\mathrm{A}_{\mathrm{ls}} \mathrm{M} / \Gamma_{\mathrm{E}} \mathrm{M}\right)$ ratio in Mammals and Aves range between 5.0 and 60 . In evolutionary aspect this ratio grows about 10 fold from Poikilotherms to Mammals and Aves and $1.0 \times 10^{4}-1.0 \times 10^{5}$ folds from Unicellular organisms to Mammals and Aves. The growing of $\mathrm{A}_{\mathrm{Is}} / \Gamma_{\mathrm{E}}$ ratio during animal evolution can be connected with 23 orders of magnitude increasing of the body mass from Unicellular organisms to Mammals and Aves, because of increasing of energy for locomotion and gravity-depending life processes during embryogenesis, growth and reproduction. For example, mechanical load is about 3 orders of magnitude larger for land-living than water-living organisms [32]. Biological system has hierarchical structure with cells, organs, organisms, layers of organisms, group and ecological system. The similar hierarchical structure exists during individual development of given organism, starting from embryogenesis to organismal growth and reproduction. Actions of gravity are caused by processes and mechanisms that are different in each layer of the hierarchy [33]. Because of this future, summing up gravitational action on cells does not explain gravity for biological system at upper layers [19]. This suggested that particular stages of animal's development, which extends from fertilization through implantation, organogenesis and post-natal maturation might be differentially sensitive to alterations in gravitation field $[34,35,36]$.

Emergent phenomena in the biosphere on Earth are undoubtedly simultaneously under influence of gravitational and heat emission of Earth and Sun. While the gravity can be 
considered as direct or indirect homeostatic factor for emergence and complexification of organisms [1] the heat emission of Earth and Sun appear direct energizing factor and play a key role in appearance of the life $[37,38]$. There is a colossal temperature difference between the Earth and Sun, but nevertheless the heat emission of Earth and Sun are complementing one another so they can keep relatively constant average temperature on Earth surface 286- 288K, necessary for existing of the biosphere [39].

The comparing of the lifespan metabolic potentials $\left(\mathrm{A}_{\mathrm{ls}}\right)$ with 'heat' potential of the Earth $\left(\mathrm{A}_{\mathrm{E}}\right)$ and the Sun $\left(\mathrm{A}_{\mathrm{S}}\right)$ shows that the metabolic potentials of Unicellular organisms $\left(1.0 \times 10^{2}-2.5 \times 10^{3} \mathrm{~kJ} / \mathrm{kg}\right)$ are smaller than 'heat' potential of the Earth $\left(\mathrm{A}_{\mathrm{E}}=7.0 \times 10^{3} \mathrm{~kJ} / \mathrm{kg}\right)$ (see Fig. 1). The 'heat' potential of the Earth divides metabolic potentials of Unicellular organisms from metabolic potentials of animals (Poikilotherms, Mammals and Aves). The full diapason of lifespan metabolic potentials $\left(3.0 \times 10^{4}-3.5 \times 10^{6} \mathrm{~kJ} / \mathrm{kg}\right)$ of animals is placed between Earth 'heat' potential $\left(\mathrm{A}_{\mathrm{E}}=7.0 \times 10^{3}\right.$ $\mathrm{kJ} / \mathrm{kg})$ and Sun 'heat' potential $\left(\mathrm{A}_{\mathrm{S}}=4.5 \times 10^{6} \mathrm{~kJ} / \mathrm{kg}\right.$. Thus, the 'heat' potentials of the Earth and the Sun limit both, from above and from bellow the diapason of the lifespan metabolic potentials of animals.

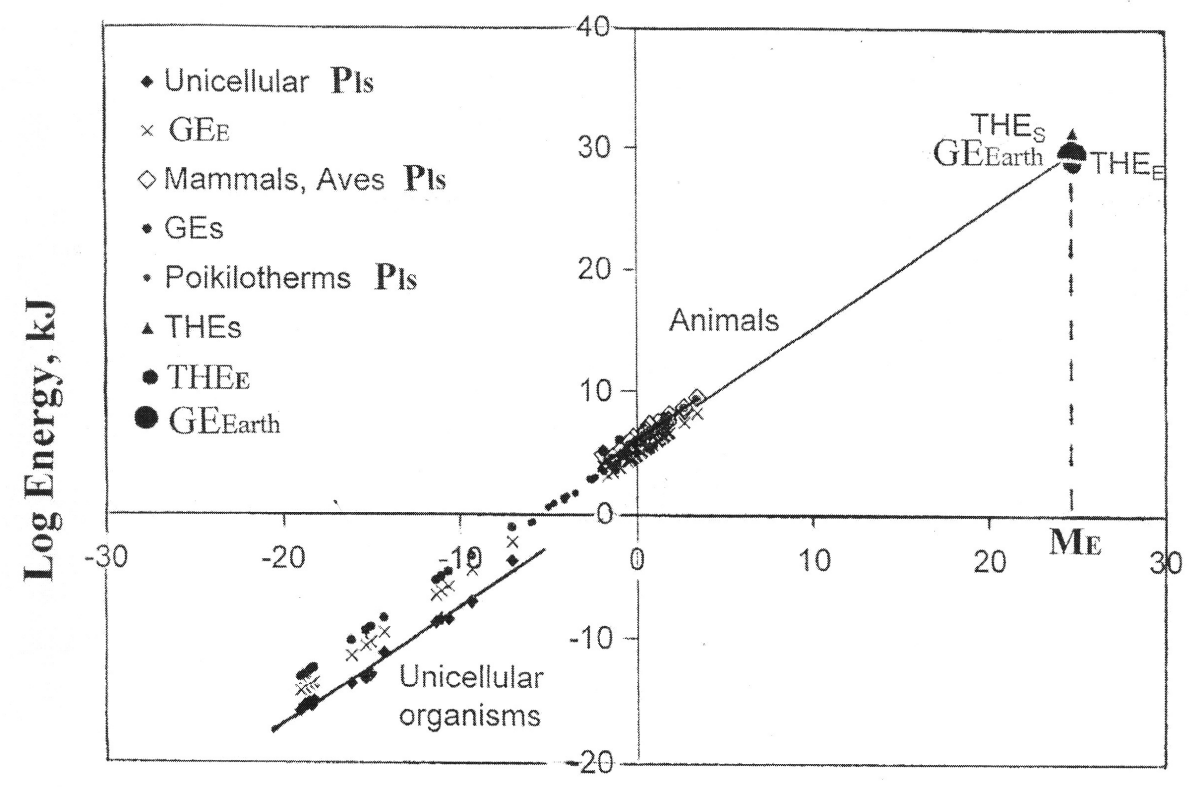

\section{Log Mass, $\mathrm{kg}$}

Figure 2. Scaling in log-log plots of energy (total metabolic energy per lifespan of Unicellular organisms, Poikilotherms, Mammals and Aves- Pls in kJ; gravitational energy of living organisms $-G E_{E}$ in $\mathrm{kJ}$; gravitational energy of Earth $-G E_{E a r t h}$ in $\mathrm{kJ}$; total heat energy of Earth $-T H E_{E}$ in $\mathrm{kJ}$ and total heat energy of Sun - THE in $k J$.

On Fig.1 the 'heat' and gravitational potentials of the Earth differs about 10 fold $\left(\mathrm{A}_{\mathrm{E}} / \Gamma_{\mathrm{E}} \approx 0.1\right)$ and limit from below lifespan metabolic potentials of Poikilotherms, Mammals and Aves. On the contrary, the 'heat' and gravitational potentials of the Sun differ $1 / 2$ fold $\left(\mathrm{A}_{\mathrm{S}} / \Gamma_{\mathrm{S}} \approx 0.5\right)$ and limit from above lifespan metabolic potentials of Poikilotherms, Mammals and Aves.

This coincidence in the location of the gravitational, 'heat' and metabolic potentials may means that gravitational and 'heat' potentials of the Earth and the Sun determined the diapason of metabolic potentials of the living organisms. However, the biological evolution is determined by the cosmological evolution of the Earth and the Sun and it can be expected the existence of resemblances, coincidences and regularities between the evolutionary characteristics of living organisms and cosmological characteristics of the Earth and the Sun.

The location of the gravitational, 'heat' and metabolic potentials of the living organisms, Earth and Sun receive specific biological sense, after scaling of all potentials and energy on the same 'mass-energy' coordinate system (Fig.2). On Fig. 2 is presented the graphical line between the total metabolic energy per lifespan of Unicellular organisms, Poikilotherms, Mammals and Aves and their body mass. On the same graphical lines are placed the data for the total heat energy $\left(\mathrm{THE}_{\mathrm{E}}=4.5 \times 10^{31} \mathrm{~J}\right)$ and gravitational energy $\left(\mathrm{GE}_{\mathrm{Earth}}\right.$ $\left.=2.238 \times 10^{32} \mathrm{~J}\right)$ of the Earth, and the total heat energy $\left(\mathrm{THE}_{\mathrm{S}}=\right.$ $2.7 \times 10^{34} \mathrm{~J}$ ) of the Sun versus body mass of the Earth $\left(\mathrm{M}_{\mathrm{E}}=5.97 \times 10^{24} \mathrm{~kg}\right)$. The imaginary lines of gravitational energy of Unicellular and Multicellular organisms in gravitational field of the Earth and the Sun on Fig. 2 are tightly placed on both sides of the graphic line of total metabolic energy per lifespan of animals. Statistical analyses have showed that nearly a linear relationship between the total metabolic energy per lifespan of Poikilotherms $\left(\mathrm{P}_{\mathrm{ls}}, \mathrm{kJ}\right)$, total heat energy $\left(\mathrm{THE}_{\mathrm{E}}, \mathrm{kJ}\right)$ of the Earth and the body mass $(\mathrm{M}, \mathrm{kg})$ of Poikilotherms and Earth $\left(\mathrm{M}_{\mathrm{E}}, \mathrm{kg}\right)$ in $\log$-log plots holds (Fig.3): 


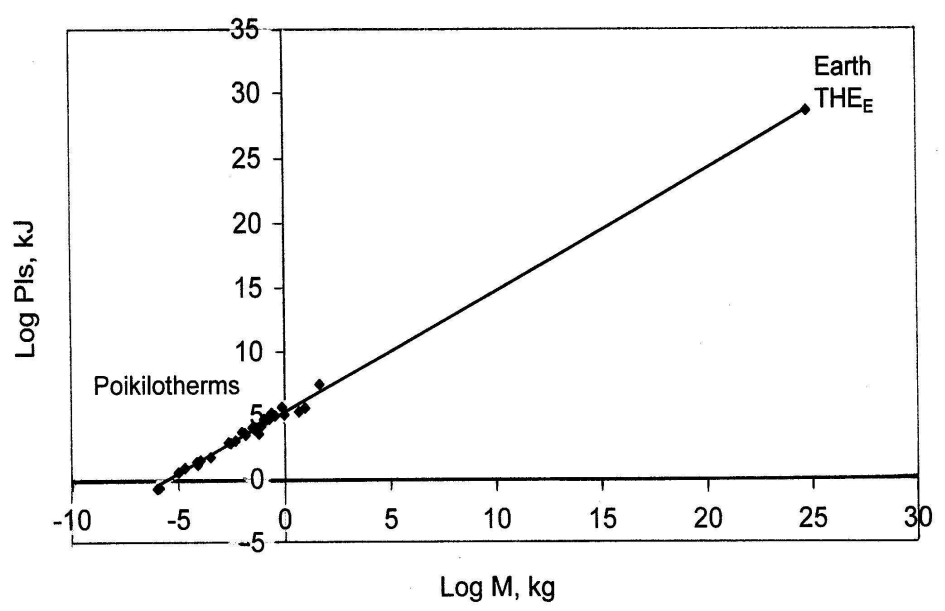

Figure 3. The relationships between the total metabolic energy per lifespan (Pls, $\mathrm{kJ})$ of Poikilotherms, total heat energy $\left(T H E_{E}=4.5 \times 10^{28} \mathrm{~kJ}\right)$ of Earth and the body mass $(M, \mathrm{~kg})$ of poikilothermic organisms, and Earth $\left(M_{E}=5.97 \times 10^{24} \mathrm{~kg}\right)$.

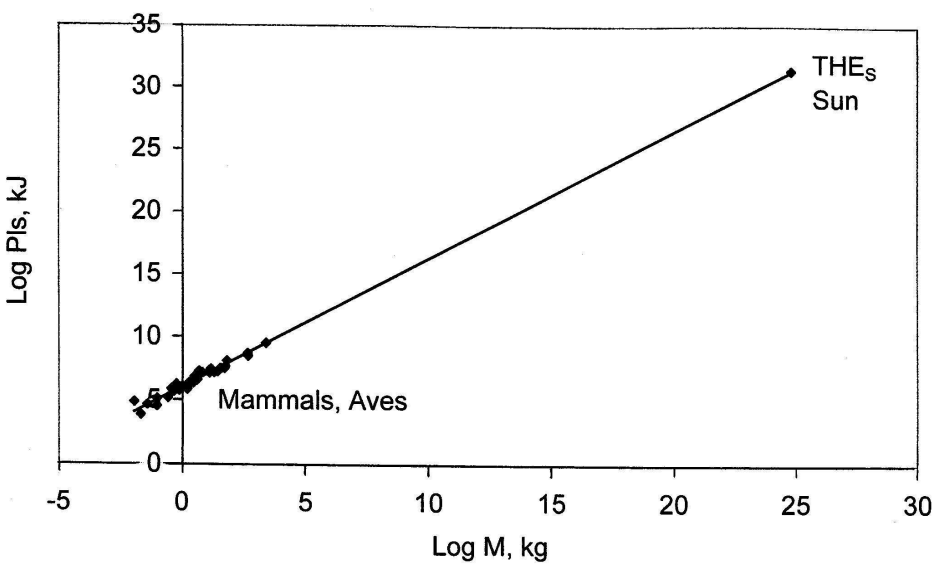

Figure 4. The relationships between the total metabolic energy per lifespan (Pls, $\mathrm{kJ})$ of Mammals and Aves, total heat energy $\left(T H E_{S}=2.7 \times 10^{31} \mathrm{~kJ}\right)$ of Sun and body mass $(M, \mathrm{~kg})$ of mammalian and avian organisms, and Earth $\left(M_{E}=5.97 \times 10^{24} \mathrm{~kg}\right)$.

$$
\mathrm{P}_{1 \mathrm{~s}}=1.696 \times 10^{5} \mathrm{M}^{0.949} \quad\left(\mathrm{R}^{2}=0.996\right)
$$

A similar relationship in log-log plots between the total metabolic energy of Mammals and Aves $\left(\mathrm{P}_{1 \mathrm{~s}}, \mathrm{~kJ}\right)$, the total heat energy of Sun $\left(\mathrm{THE}_{\mathrm{S}}, \mathrm{kJ}\right)$, and body mass $(\mathrm{M}, \mathrm{kg})$ of Mammals, Aves and Earth ( $\left.\mathrm{M}_{\mathrm{E}}, \mathrm{kg}\right)$ holds (Fig.4):

$$
\mathrm{P}_{1 \mathrm{~s}}=10.2 \times 10^{5} \mathrm{M}^{1.023} \quad\left(\mathrm{R}^{2}=0.996\right)
$$

Near to 1.0 corresponding coefficient $\mathrm{R}^{2}$ in equations (11-12) show that these relationships are not random. Accordingly relationship (11) the data for the total metabolic energy of Poikolthermic animals, the total heat energy emitted from the Earth and corresponding body mass lies on the same line, while accordingly relationship (12) the data for the total metabolic energy of Mammals and Aves, total heat energy emitted from Sun on Earth and corresponding body masse lies on one and same line too.

A similar relationship was not observed in unicellular organisms, since the appearance of the first primitive forms of bacterial life before 3850 million years ago is connected with the Earth only [40]. The earliest primitive organisms (archaea and bacteria) have received both, matter and energy from hydrothermal vents of Earth and didn't need solar energy [38].
The appearance of the Poikilothermic organisms (700 million years ago) and the appearance of Mammals and Aves (230-175 million years ago) coincide approximately with the time when the total heat energy of the Earth and the Sun reached certain values, satisfying the relationships (11) and (12). This is an indication that the thermal evolution of the Earth and the Sun determines the rate and time of appearance of living organisms during their biological evolution.

Moreover, the gravitational and heat energy of the Earth and the Sun differ from each other in relation to their biological significance and influence on the living organisms. While the gravity 'consumes' the constant amount of metabolic energy, necessary for overcomes of their influence, the heat energy of the Earth and the Sun 'creates' metabolic energy trough the chemosynthesis, photosynthesis and food chain of organisms in the biosphere.

As the total heat energy, radiated from the Earth and the Sun increases during time, this should leads to the increasing of the total metabolic energy of the living organisms during their evolution in time, accordingly relationships (11) and (12). This shows that the heat emission of the Earth and the Sun appears to be the main driving force for animal evolution, in comparison to the constantly acting gravitational force. 
Nearly the same values of the 'heat' and gravitational potentials of the Earth and the Sun show that the complex arranged animals with large body mass appear only when the accumulated total heat energy (per Earth lifespan) in Earth's biosphere is sufficient to balance the gravitational energy of the Earth and the Sun on ground. This requires approximately the equality between the two energies, gravitational and heat, from the origin of the Earth up to appearance of the complex arranged animals with large body mass.

In conclusion, the present investigations show that the evolution and mass-energy characteristics of the living organisms on the Earth depend simultaneously on the evolution and mass-energy characteristics of the Earth and the Sun.

These allow us to predict how the same characteristics of living organisms will depend in case, if they grow on other planet or Cosmic System, different from Solar System.

\section{References}

[1] N. Fujiwara, "The scaling rule for environmental organizing systems in a gravitational field," BioSystems, vol. 73, pp. $111-116,2004$

[2] G. Clément, "Introduction to space biology," The Space Technology Library, vol. 18, pp. 1-50, 2006.

[3] R.J. Blakely, Potential theory in gravity and magnetic applications. Cambridge University Press, UK, 1996.

[4] B.K. McNab, "On the utility of uniformity in the definition of basal rate of metabolism,” Phys. Zoology, vol.70, pp.718-720, 1997.

[5] A.T. Atanasov, "The linear allometric relationship between total metabolic energy per life span and body mass of Poikilothermic animals," Biosystems, vol. 82, pp. 137-142, 2005.

[6] A.T. Atanasov, "Linear allometric relationship between total metabolic energy per lifespan and body mass of terrestrial mammals in captivity,” BJVM, vol. 9, pp. 159-174, 2006.

[7] A.T. Atanasov, "The linear allometric relationship between total metabolic energy per life span and the body mass of mammals," Biosystems, vol. 90, pp. 224-233, 2007.

[8] A.T. Atanasov, "The near to linear allometric relationship between total metabolic energy per life span and the body mass of Nonpasseriformes birds," BJVM, vol. 10, pp. 235-245, 2007.

[9] N. Fujiwara, "Origin of the scaling rule for fundamental living organisms based on thermodynamics," BioSystems, vol. 70, pp $1-7,2003$

[10] J.R. Hickey, B.M. Alton, H.L. Kyle and D. Hoyt, "Total solar irradiance measurements by ERB/NUMBUS 7. A review of nine years," Space Sci. Rev., vol. 48, pp. 321-342, 1998.

[11] A.J. Colozza, Effect of data and location on maximum achievable altitude for a solar powered aircraft. NASA Contractor Report 202326. NYMA, Inc. Brook Park, Ohio, 1997.
[12] C. Gueymard " "The sun's total and spectral irradiance for solar energy applications and solar radiation models," Solar Energy, vol. 76, pp. 423-453, 2003.

[13] H.N. Pollack, S.J. Hurter and J.R. Johnson, "Heat flow from the Earth's interior: analysis of the global data set," Reviews of Geophysics, vol. 31(3), pp. 267-280, 1993.

[14] J.G. Sclater, C. Jaupart and D. Galson, "The heat flow through oceanic and continental crust and the heat loss of the Earth," Reviews of Geophysics and Space Physics, vol. 18(1), pp. 269-311, 1980.

[15] G. F. Davies, "Thermal histories of convective Earth models and constrains on radiogenic heat production in the Earth," $\mathrm{J}$. Geophys. Res., vol. 85, pp.2517-2530, 1980.

[16] E. M. Galimov, "Redox evolution of the Earth caused by a multi-stage formation of its core," EPSL, vol. 233, pp. 263-276, 2005.

[17] H-H. Ku and R. Sohal, "Comparison of mitochondrial pro-oxidant generation and anti-oxidant defences between rat and pigeon: possible basis of variation in longevity and metabolic potential," Mech. Ageing. Dev., vol. 72, pp. 67-76, 1993.

[18] D. Volkmann and A. Sievers, "Research under reduced gravity. Part I: Bases of gravitational biology," Die Naturwissenschaffen, vol. 79, pp. 68-74, 1992.

[19] M. Yamashita and S.A. Baba, "Biology of size and gravity," Biol. Sci. Space, vol. 18, pp. 13-27, 2004.

[20] D. Valev, "Determination of total mechanical energy of the universe within the framework of Newtonian mechanics," In “arXiv:0909.2726vl [physics.gen-ph] 15 Sep 2009".

[21] P. Todd, "Gravity-depending phenomena at the scale of the single cell," ASGSB Bull., vol. 2, pp. 95-113, 1989.

[22] P. Bouloc P and R. D'Ary, "Escherichia coli metabolism in space,” J. Gen. Microbiol., vol. 137, pp. 2839-2843, 1991.

[23] S. Deguchi, H. Shimoshige, M. Tsudome, S-A. Mukai, R.W. Corkery RW, S. and K. Horikoshi, "Microbial growth at hyperaccelerations up to $403627 \times$ g," PNAS, Early Ed., 2010 (www.pnas.org/cgi/doi/10.1073/pnas.1018027108).

[24] Y. Kato, Y. Mogami and S.A. Baba, "Responses to hypergravity in proliferation of Paramecium tetraurelia," Zoology Science, vol. 20, pp.1373-1380, 2003.

[25] A.L. Demain and A. Fang, "Secondary metabolism in simulated microgravity," Chem. Rec., vol. 1, pp. 333-346, 2001.

[26] C.A. Nickerson, C.M. Ott, J.W. Wilson, R. Ramamurthy and D.L. Pierson, "Microbial responses to microgravity and other low-shear environments," Microbiol. Mol. Biol. Rev., vol. 68, pp. 345-361, 2004.

[27] B. Purevdorj-Gage, K.B. Sheehan and L.E. Hyman, "Effects of low-shear modeled microgravity on cell function, gene expression and phenotype in Saccharomyces cerevisiae," Appl. Environ. Microbiol., vol. 72, pp. 4569-4575, 2006.

[28] G. Horneck, D. Klaus and L. Mancinelli, "Space Microbiology," Microbiol. Mol. Biol. Rev., vol.74, pp. 121-156, 2010 . 
[29] V.A. Kotov, "Solar System, exoplanets and anthropic principle,” Bull. Crim. Astr. Obs., vol.105, pp. 119-128, 2009.

[30] R.A. Kerr, "Putting a Lid on Life on Europe," Science, vol. 294, pp. 1258-1259, 2001.

[31] D. Schulze-Makuch and L.N. Irwin, "Energy Cycling and Hypothetical Organisms in Europa's Ocean," Astrobiology, vol.2, pp.105-121, 2002.

[32] D. Volkmann and F. Baluska, "Gravity: one of the driving forces for evolution," Protoplasma, vol. 229, pp. 143-148, 2006.

[33] A.H. Brown, "From gravity and the organism to gravity and the cell," ASGSB Bull., vol. 4, pp. 7-18, 1991.

[34] M.D. Ross, "The influence of gravity on structure and function of animals," Adv. Space Res., vol. 4, pp. 305-314, 1984.

[35] J.R. Keefe, Final report of the NASA Mammalian Developmental Biology Working Group. In: NASA Developmental Biology Workshop (K.A. Souza and T.W.
Halstead, eds.), pp.46-63. NASA TM-86756, Washington, D.C., 1985.

[36] D. J. Wolgemuth and A.K. Murashov, "Models and molecular approaches to assessing the effects of the microgravity environment on vertebrate development," ASGSB Bull., vol. 8, pp. 63-71, 1995.

[37] K. Michaelian, "Thermodynamic stability of ecosystems," J. Theoret. Biol., vol. 237, pp. 323-335, 2005.

[38] K. Michaelian, "Thermodynamic origin of life," In "arXiv:0907.0042v3 [physics.gen-ph] 8 Sep 2010".

[39] C. Sagan and G. Mullen, "Earth and Mars: Evolution of atmospheres and surface temperature," Science (New Series), vol. 177, pp. 52-56, 1972.

[40] C.L. Van Dover, The Ecology of Deep-Sea Hydrothermal Vents. Copyright (C) 2000 by Princeton University Press, UK, 2000.

[41] J. D. Fix, ASTRONOMY: Journey to the cosmic frontiers. First Ed., University of Iowa, Mosby-Year Book, Inc., 1995. 